\title{
Successful treatment of diffuse panbronchiolitis in a child from Western China: A case report
}

\author{
NA-NA ZHAO ${ }^{1 *}$, HUI CAO ${ }^{2 *}$, SHI-SI ZHANG ${ }^{1}$ and GUO-QIANG CAO ${ }^{1}$ \\ Departments of ${ }^{1}$ Respiratory Medicine and ${ }^{2}$ Radiology, Institute of Surgery Research, \\ The Third Affiliated Hospital, The Third Military Medical University, Chongqing 400042, P.R. China
}

Received April 20, 2016; Accepted November 24, 2016

DOI: $10.3892 /$ etm.2017.4196

\begin{abstract}
The present report describes a case of diffuse panbronchiolitis (DPB) in a child from Western China and the favorable outcome associated with early diagnosis. DPB is an uncommon presentation in pediatric patients. A 13-year-old Chinese boy was admitted to the respiratory outpatient department due to recurrent cough and progressive exertional dyspnea that had persisted for 1 year. An initial diagnosis of bronchial asthma was made, and the patient was prescribed inhaled fluticasone combined with salmeterol (50/250 $\mu \mathrm{g}$, twice daily), and montelukast (4 mg daily). However, 2 months later no clinical improvement was observed. The disease was re-diagnosed as DPB following the identification of features such as centrilobular small nodular opacities, a 'tree-in-bud appearance' and thickening of the bronchial walls meeting the diagnostic criteria for DPB. Complete resolution of the disease and sustained alleviation of the patient's respiratory symptoms were achieved following the early institution of erythromycin therapy, and the exacerbation of chronic bronchitis was reduced. In conclusion, it is essential to consider that successful treatment for DPB lies in early diagnosis and early treatment. DPB may be treated well by use of erythromycin.
\end{abstract}

\section{Introduction}

Diffuse panbronchiolitis (DPB) is an idiopathic inflammatory disease characterized by chronic respiratory bronchioles of the bilateral lung that is effectively treated using macrolides (1), and has historically been associated with a very poor prognosis. Clinically, DPB is characterized by persistent

Correspondence to: Professor Guo-Qiang Cao, Department of Respiratory Medicine, Institute of Surgery Research, The Third Affiliated Hospital, The Third Military Medical University, 10 Changjiangzhilu, Daping, Yuzhong, Chongqing 400042, P.R. China

E-mail: cao_guoqiang@yeah.net

*Contributed equally

Key words: diffuse panbronchiolitis, child, Western China cough, sputum and progressive exertional dyspnea. DPB is a progressive suppurative and obstructive airway disease, resulting in bronchiectasis, respiratory failure and mortality, if left untreated (2). This disease has been observed in the Japanese population, and the incidence of DPB is high among Asian individuals, the incidence was calculated at $\sim 1$ per 10,000 individuals per year. The average age of onset is 40 years, however, it is rare in children (3). The exact pathogenesis of this disease is still unknown but it may be related to infection and genetic factors. The diagnostic criteria of DPB was as follows: i) A persistent cough, sputum and exertional dyspnea, ii) history or current symptoms of chronic sinusitis, iii) bilateral, diffuse, small nodular shadows on a plain chest radiograph or centrilobular micro nodules on chest computed tomography (CT) images, iv) coarse crackles, v) the ratio of forced expiratory volume in $1 \mathrm{sec}$ (FEV1) to forced vital capacity (FVC) (FEV1/FVC) $<70 \%$ and partial pressure of oxygen $\left(\mathrm{PaO}_{2}\right)<80 \mathrm{mmHg}(10.64 \mathrm{kPa})$ and vi) cold agglutinin CHA titer of $\geq 64$ (4). Long-term low dose therapy with macrolide antibiotics has been demonstrated to significantly improve the survival of DPB patients (2). To the best of our knowledge, DPB has not been reported in a child from Western China. A 13-year-old Chinese girl was described as the first child in the Chinese literature with diffuse panbronchiolitis from northern China (5). The present study describes a case of DPB in a Chinese boy that was initially misdiagnosed as bronchial asthma.

\section{Case report}

A 13-year-old Chinese boy was admitted to the respiratory outpatient department of The Third Affiliated Hospital of Third Military Medical University (Chongqing, China) in November 2014 with the chief complaint of recurrent cough and progressive exertional dyspnea that had persisted for 1 year. Ethical approval was obtained from the Institutional Review Board of the Institute of Surgery Research, Daping Hospital, Third Military Medical University and signed informed consent was obtained from the parents of the patient.

The patient had a history of a slight cold over the previous 2 weeks. He had no family history of asthma and was a non-smoker. A few moist rales and expiratory rhonchi were audible bilaterally on basilar areas of the back on chest auscultation. No clubbing of the fingers was observed. Lung function 

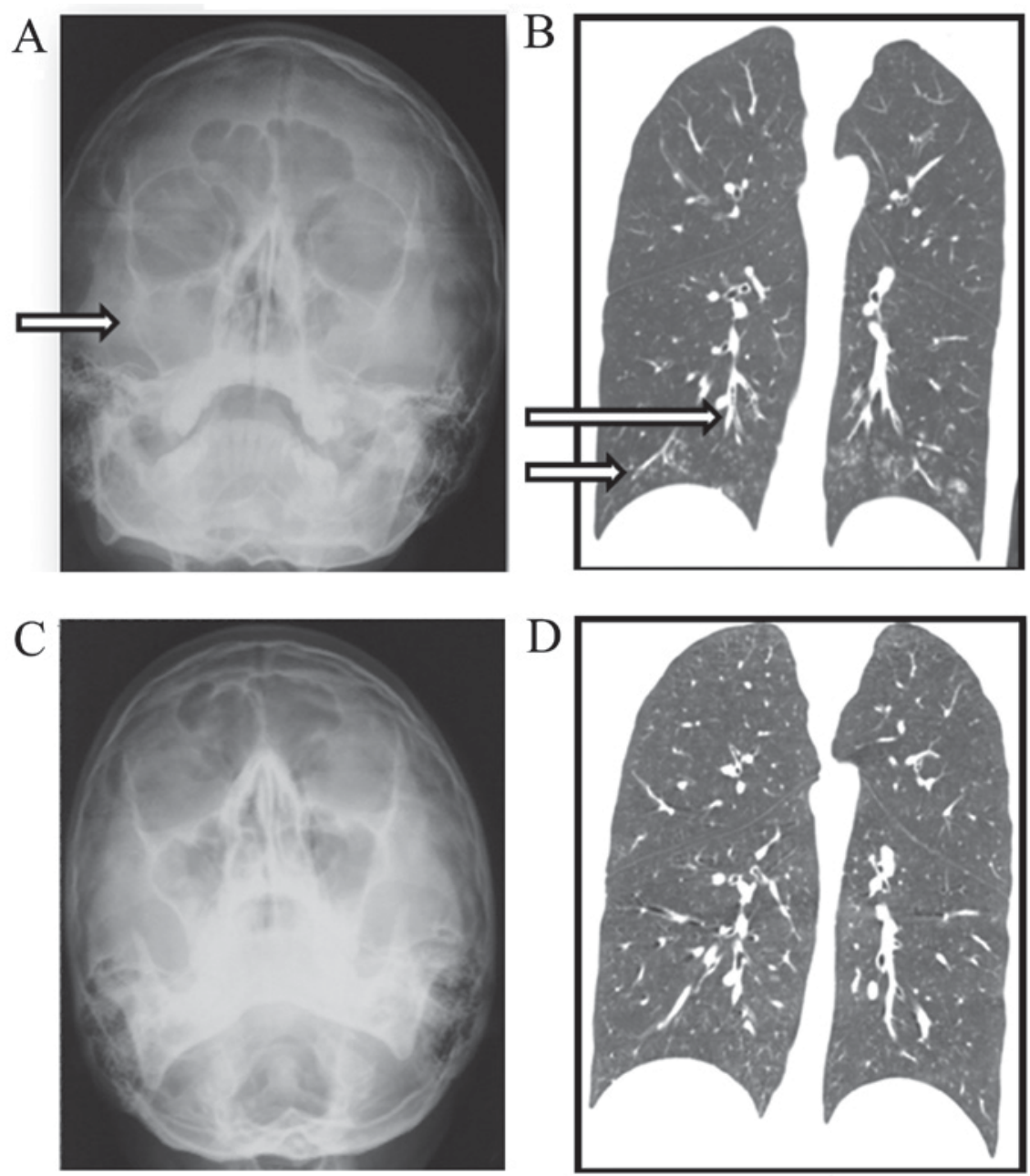

Figure 1. X-ray and chest HRCT images of the patient. (A) X-ray image prior to the diagnosis of DPB showed that the maxillary sinus was affected by proliferation of the annular mucosa without fluid pooling (arrow), and the sinus cavity wall exhibited no bone destruction or absorption phenomenon. (B) HRCT image prior to DPB diagnosis revealed centrilobular small nodular opacities, a 'tree-in-bud' appearance and thickening of the bronchial walls (arrows). (C) X-ray image taken 1 year following cessation of erythromycin therapy showing no evidence of fluid in the sinuses, and mild thickening of the mucous membrane. (D) HRCT of the thorax indicated no nodular infiltrate or bronchiectasis. HCRT, high-resolution computed tomography; DPB, diffuse panbronchiolitis.

testing revealed a forced expiratory volume 1 /forced vital capacity ratio of $<70 \%$ (normal ratio is $\geq 70 \%$ ), and arterial blood gas testing revealed an arterial partial pressure of oxygen $\left(\mathrm{PaO}_{2}\right)$ of $<80 \mathrm{mmHg}$ (normal $\mathrm{PaO}_{2} \geq 80 \%$ ). The patient was diagnosed with bronchial asthma, and was prescribed inhaled fluticasone combined with salmeterol (50/250 $\mu \mathrm{g}$, twice daily; GlaxoSmithKline plc. London, UK), and montelukast (4 mg daily, oral administration, Merck \& Co., Inc., Whitehouse Station, NJ, USA). Regular outpatient department follow-up was recorded.

Following 2 months of treatment, no clinical improvement was observed and the patient's physiology worsened. The boy had a cough productive of purulent sputum, particularly in the morning, and had an extremely nasal voice. Thus, Water's view X-ray scanning was performed. Bilateral maxillary sinusitis was indicated (Fig. 1A). X-ray imaging revealed proliferation of the annular mucosa, but no fluid pooling. Also, the sinus cavity wall exhibited no bone destruction or absorption phenomenon, which prompted chronic inflammation of the two maxillary sinuses. The clinical features of this case were as follows: Persistent cough, sputum and exertional dyspnea, expiratory rhonchi and moist rales audible bilaterally on basilar areas of the back, chronic sinusitis, and spirometrically detected airway obstruction that did not improve with the use of a bronchodilator. Therefore, a preliminary diagnosis of DPB was made. Chest high-resolution computed tomography (HRCT) revealed centrilobular small nodular opacities, a 'tree-in-bud appearance' and thickening of the bronchial walls (Fig. 1B).

On the basis of this clinical profile, the child was diagnosed with DPB. The initial treatment protocol was combination therapy with erythromycin $(0.5 \mathrm{~g}$, oral administration, once a day; Wanhe Pharmaceutical Co., Ltd., Tianchang, China) and inhaled fluticasone combined with salmeterol $(50 / 250 \mu \mathrm{g}$, 1 inhalation twice a day). The adjuvant therapy with inhaled salmeterol/fluticasone was administered in order to relieve the cough and dyspnea. After 4 weeks of treatment, the presenting symptoms of dyspnea on exertion, cough and nasal congestion had improved or stabilized. The fluticasone/salmeterol therapy was then stopped. A subsequent maintenance treatment with erythromycin at a dose of $0.5 \mathrm{~g}$ every other day was continued for 1 year. During that time, the symptoms and results of imaging and pulmonary function tests progressively improved, and remained normal for 1 year following cessation of the erythromycin therapy (Fig. 1C and D). 


\section{Discussion}

The diagnosis of the present case was made using previously reported diagnostic criteria for DPB (6). DPB was first described in China in 1996, and the average age of onset is 40 years (7). We found by only 3 previous reports of DPB cases in children. In 2007, a 13-year-old Chinese girl was the first child to be described with DPB in the Chinese literature, indicating that DPB morbidity may affect children. The girl who was diagnosed with diffuse panbronchiolitis following a 12-year history of progressive course of cough with purulent sputum and chronic sinusitis (5). A 12-year-old Caucasian Turkish girl of first-degree consanguineous parentage was described as the first child in the English language literature with diffuse panbronchiolitis. She was diagnosed with diffuse panbronchiolitis after a 5 -year history of productive cough with purulent sputum and chronic sinusitis (8). A 10-year-old South Korean boy who was adopted by non-smoking professional American parents as a 4-month-old infant was described as the youngest child with diffuse panbronchiolitis and uniquely provides the results of a bronchoalveolar lavage. He had a slowly progressive course over a 6-month period (3). Each of the 3 patients in these reports was initially misdiagnosed, with nasosinusitis, bronchiectasis or even pulmonary tuberculosis as incorrect diagnoses. The duration of misdiagnosis ranged from 6 months to 12 years. None of these children were diagnosed correctly at the first instance.

The delay time interval between onset and diagnosis in the present case was the shortest in a child case of DPB reported to date. Additionally, to the best of our knowledge, this case is the first child with DPB reported in Western China. In the present patient, through early diagnosis and intervention with erythromycin, complete reversal of the signs of the disease without evidence of persistent respiratory compromise was achieved.
This was in contrast to the 3 previously reported cases in children, who had presented with substantial, irreversible lung disease, which continued to deteriorate, despite treatment with macrolide. A prolonged period of diffuse panbronchiolitis is associated with progressive lung damage, so this case illustrates the importance of early diagnosis and treatment.

In conclusion, DPB may be able to affect children anywhere in Mainland China; therefore, it is recommended that respiratory physicians and pediatricians are familiar with the clinical and radioactive features of DPB. In children who have chronic cough, expectoration, gasping, decreased pulmonary ventilation function and $\mathrm{PaO}_{2}$, and nasosinusitis, either at presentation or in their medical history, this disease should be considered and HRCT used to obtain an early diagnosis so that treatment with macrolide is initiated as early as possible.

\section{References}

1. Sugiyama Y: Diffuse panbronchiolitis. Clin Chest Med 14: 765-772, 1993.

2. Sugimoto S, Miyoshi K, Yamane M and Oto T: Lung transplantation for diffuse panbronchiolitis: 5 cases from a single centre. Interact Cardiovasc Thorac Surg 22: 679-681, 2016.

3. Weinberger M, Fischer A and Kao S: Diffuse panbronchiolitis in a 10-year-old boy. Pediatr Pulmonol 50: E32-E34, 2015.

4. Chuang MC, Chou YT, Lin YC, Hsieh MJ and Tsai YH: Diffuse panbronchiolitis-The response and recurrence after erythromycin therapy. J Formos Med Assoc 115: 876-882, 2016.

5. Zhao SY, Peng Y, Zhou CJ, Jiao Ax and Jiang ZF: Diffuse panbronchiolitis in a child: Case report and literature review. Zhonghua Er Ke Za Zhi 45: 504-507, 2007 (In Chinese).

6. Kudoh S and Keicho N: Diffuse panbronchiolitis. Clin Chest Med 33: 297-305, 2012.

7. Liu YN, Hu H and Cai ZL: A case of diffuse panbronchiolitis. Chin J Tuberc Respir Dis 19: 118-119, 1996 (In Chinese).

8. Asian AT, Ozcelik U, Talim B, Haliloglu M, Dogru D, Dalgic F and Kiper N: Childhood diffusepanbronchiolitis: A case report. Pediatr Pulmonol 40: 354-357, 2005. 